\title{
A Tale of Three Chinese Societies: The Quality of Life and Well-Being of Chinese People in a Changing World
}

\author{
Po-Keung Ip • Daniel T. L. Shek
}

Accepted: 5 July 2013 / Published online: 12 July 2013

(C) Springer Science+Business Media Dordrecht 2013

Quality of life (QOL) and well-being (WB) as research topics have received much attention in the Western literature. Researchers in different disciplines, including medicine, nursing, rehabilitation science, psychology, sociology, social work, business and philosophy have conducted research in the broad fields of quality of life and well-being. Nevertheless, the notions of quality of life and well-being are different, though they share some conceptual overlaps. Quality of life refers to mainly the social aspects of life that go beyond economic life of people in a society. Historically, because the social aspect of life is the major focus of concern, the measures involved were aptly denoted as "social indicators". It is a broad measure of life in general that include its economic, social, environmental, health, and cultural aspects. What aspects or parameters of life should be included in the quality of life depends very much on how important these aspects or parameters are regarded as constitutive of a good life, and is highly dependent on how a society's values and priorities. Well-being of a country refers to its people's perception of life satisfaction about various domains of living. When the concept is used in this way, it means the subjective well-being of people of a country. It is subjective because the perception is expressed as a personal subjective judgment of life satisfaction. Subjective wellbeing can be measured by either the prevalence of positive affect with respect to different domains, or the extent of fulfillment of life goals or values of people, depending on whether well-being is conceived hedonically or in the eudaimonic manner.

In contrast to the abundance of QOL and WB research in the West, related research is grossly inadequate in different Chinese societies (Shek et al. 2005; Shek 2010). Based on the search term "quality of life", computer search up to June 2013 showed that there were 41,479 citations in PsycINFO, 6,720 citations in Sociological Abstracts, and 32,881 citations in Medline. In contrast, when we used "quality of life" and "Chinese" as the search

P.-K. Ip (ه)

Graduate Institute of Philosophy, National Central University, Zhongli, Taiwan e-mail: pkip@cc.ncu.edu.tw

D. T. L. Shek

Department of Applied Social Sciences, The Hong Kong Polytechnic University, Hung Hom, Hong Kong 
terms, identical search showed that there were only 995, 184 and 604 citations in PsycINFO, Sociological Abstracts, and Medline, respectively. Similarly, Based on the search term "well-being", computer search up to June 2013 showed that there were 50,642 citations in PsycINFO, 16,649 citations in Sociological Abstracts, and 11,446 citations in Medline. In contrast, when we used "well-being" and "Chinese" as the search terms, identical search showed that there were only 1,106, 405 and 219 citations in PsycINFO, Sociological Abstracts, and Medline, respectively. These figures strongly indicate that additional effort is much needed to conduct quality of life and well-being research in different Chinese societies. However, it should be noted that the term "Chinese" is used in a cultural, but not political sense. Chinese societies are societies that basically share and live the Chinese culture. Thus, Chinese societies can have different political and economic systems, of which Hong Kong, Macau, Taiwan and China in Greater China are good examples.

Since the late 1980s, research related to quality of life, social indicators or well-being have started in Greater China, a region comprising China, Taiwan, Hong Kong and Macau (for details, see Ip \& Cheung in this issue). Similar studies began to emerge over the first decade of 2000. In China, studies included cross-province well-being surveys published in Chinese gave valuable information about how Chinese people perceived their happiness. Studies published in the English literature offered information about, among others, the relationship between rising standard of living and the well-being, as well as life satisfaction of Chinese people. In Taiwan, there have been increasing efforts in the study of quality of life and well-being. Many of these studies conducted in the last decade or so used concepts or measures straightly taken from the West with little or no adaptation. There are little studies using a framework of well-being which is specifically constructed for the region. This way of conducting well-being research may create problems. Despite similarities between human societies, there are admittedly discernable differences between societies in different cultural regions. Greater China, as a region with its unique cultural and economicpolitical conditions, is different from countries which share a dominant Anglo-Saxon culture (e.g. The US, UK and Australia). However, recent studies of Chinese well-being or quality of life, as well as well-being in Asian societies (for details see Ip, in this issue) often used concepts and/or measures largely developed in the West. These measures are useful in providing a general picture of well-being of a society, or for international comparisons. However, If one seeks a more nuanced and deeper understanding of the wellbeing of a particular society, the culture and preferences of that society should be taken seriously as people's happiness are deeply influenced by the cultures and values they adopt. Thus it is legitimate to ask whether the concepts and measures developed mainly in the West can be properly used to understand the well-being of societies in the East. Consequently, there is a need to examine the possibility of alternative frameworks and measures to this effect.

There are several justifications why more Chinese QOL and WB research should be conducted. First, the size of the Chinese population speaks for the importance. The World Population Clock (2013) showed that the population of China was 1.35 billion in 2013, which constituted roughly one-fifth of the world's population in 2013. Obviously, to understand and test the generalizations of any Western theories on quality of life, QOL and WB researchers must collect data from Chinese people. At the same time, Chinese data will enable QOL and WB researchers to construct new models on QOL which provide theoretical insight for Western QOL and WB researchers.

The second reason why QOL and WB studies should be conducted in China is that China has a history of more than 5,000 years, with different cultural conceptions of "good 
life" and traditions influencing the QOL and WB of contemporary Chinese people. In contrast to the emphases on individual choices and expression of emotions, collective interests and self-cultivation were emphasized whereas hedonism, individual desires, and emotional expression were deemphasized in traditional Chinese culture. Obviously, these traditional cultural roots would have important impact on Chinese people.

The third reason for conducting more Chinese QOL and WB studies is that there are unique and changing socio-cultural conditions influencing the quality of life of people in different Chinese societies. In mainland China, the political system is the fundamental determinant of the quality of life in Chinese people. As a Communist regime, the national goal to build up a "socialist country with Chinese characteristics" has shaped the quality of life of individuals, families, communities and society at large. Before the open door policy in the late 1970s, freedom of speech was not allowed and life choices were limited in mainland China. Political struggles such as the Cultural Revolution have affected billions of lives. Even after more than 30 years of economic reform, the State is still very strict in her control of the media (such as blocking sensitive Internet sites), although it must be admitted that individual freedom and flow of information have increased much in the past three decades. Within the socialist political regime, the overriding importance of the State is emphasized in Communist China and the State's interest is always above the interest of individuals and families. One good example is the "one-child policy" where families will be punished if they have more than one child. Furthermore, with the worsening of corruption and widening of the rich and poor gap, these changing socio-cultural conditions definitely influence the quality of life Chinese people in mainland China.

Taiwan, formally known as The Republic of China, is an island state with 23 million inhabitants the majority of which is Han Chinese whose ancestors migrated to the island from China since the Ming-Qing dynasties. Famous for its high-tech industry and beautiful scenery and varied landscape, Taiwan is the only Chinese society where citizens can directly elect their presidents and legislators in open and free elections in its newly developed democracy while preserving much of traditional Chinese culture. As well as being a middle-income country, its export-oriented economy has been experiencing low growth and difficulties in structural adjustment since the financial crisis which makes unemployment or underemployment a particularly challenging issue for the government. The society is now experiencing two demographic challenges: low fertility and rapid demographic ageing of the population which have profound impact on the future of Taiwan's economy, society, education, health care and welfare, among others. The tense relationship between China and Taiwan since the mid 90s has been eased off and substantially improved since the Kuomintang (KMT) regained power in 2008. However, to maintain peaceful and trusting cross-strait relationship has always been an enduring challenge for the two governments.

In the case of Hong Kong, there are several unique features that should be noted by quality of life researchers. First, compared to mainland China and Taiwan, Hong Kong is most Western because it was a British colony for more than 150 years before 1997 . Second, competition in Hong Kong is very strong, thus creating much stress for people in Hong Kong. Third, the cost of living in Hong Kong is very high because of the rising property price but stagnant wages. Fourth, the gap between the rich and poor has widened much after 1997. For example, one out of four adolescents is experiencing economic disadvantage. Fifth, political arguments are very common after handover and dissatisfaction with the Hong Kong Government of the Special Administrative Region has increased tremendously. 
Based on a review of the published QOL and WB studies and the three existing projects (Indicators of Social Development Project, Social Development Index and the CUHK Hong Kong Quality of Life Index), Shek (2011) highlighted several observations with respect to the quality of life of Hong Kong. These include: (a) there has been progress in the quality of life in Hong Kong after 1997; (b) despite overall progress in quality of life in Hong Kong, some social groups do not show much improvement; (c) subjective quality of life has dropped after 1997; (d) warning signals about declining quality of life in Hong Kong are emerging; (e) there are many psychosocial correlates of subjective quality of life; (f) quality of life in Hong Kong is better than quality of life in China based on some indicators; and (g) there are conflicting pictures based on different studies. Finally, several methodological issues pertinent to the study of quality of life in Hong Kong are outlined which provide some food for thoughts regarding the future direction of quality of life research in Hong Kong.

The preceding discussion highlights two key systems of influence on Chinese quality of life in different Chinese societies-shared traditional Chinese cultural influence as well as the unique and changing economic-politico-socio-cultural forces within and between them. Within this framework, many QOL and WB questions remain to be answered. The fundamental question is a descriptive one-what is the nature of quality of life and well-being in different Chinese societies within the changing economic, social and political contexts? In particular, whether quality of life in Chinese people varies across different Chinese societies is an interesting question to be asked. Unfortunately, QOL and WB studies in different Chinese societies are still very piecemeal and scattered. Besides variation of QOL and WB across Chinese societies, it is also important to ask whether quality of life of Chinese people varies across time as well. For example, Shek et al. (2005) and Shek (2010) pointed out that there are few Chinese studies on quality of life among Chinese people throughout the life span. Hence, it would be important to ask whether quality of life of Chinese adolescents varies across their adolescent years. The well-being of Chinese societies can be comparatively understood from a cross-cultural perspective (Ip 2009). Besides the generalizability of findings across Chinese societies and time, it is also important to ask whether QOL and WB research findings can be generalized across different populations as well. For example, in view of the growing problem of poverty in the global context, it is necessary to conduct more research on the quality of life in people experiencing economic disadvantage. Finally, besides individual QOL and WB, family quality of life is also very important for Chinese people. Unfortunately, as pointed out by Shek (2010), there is a lack of studies on family quality of life. Hence, more studies should be done.

Against this background, the papers in this special issue on Chinese societies represent an excellent effort to provide some answers to the above puzzles. Xing and Huang's paper: "The relationship between age and subjective well-being: evidence from five capital cities in mainland China," explored the relationship between age and subjective well-being using a large sample collected from five cities in mainland China. Although the findings provided partial support for the U-shape relationship between age and subjective well-being, it was also found that different types of relationship were revealed by different measures of subjective well-being. While some findings are consistent with those in the literature, unique findings also emerged from the findings.

The three papers from Hong Kong are based on a longitudinal data set involving junior secondary school students. In response to the observation that there are few studies on QOL in Chinese adolescents experiencing economic disadvantage, Shek and Lin's paper: "Personal well-being and family quality of life of early adolescents in Hong Kong: do economic disadvantage and time matter?" presented longitudinal research findings collected from a 
large sample of junior secondary school students to examine the differences in personal wellbeing and family quality of life in Chinese adolescents with and without economic disadvantage and the related trends over time. Compared with adolescents without experience of poverty, poor adolescents showed poorer positive youth development and perceived family functioning. Besides, significant interaction effect of poverty and time on substance abuse was observed. The findings suggest that positive youth development and perceived family functioning dropped but risk behavior increased over the years.

As there are few studies on the trends and correlates of adolescent life satisfaction, Shek and Liu's paper: "Life satisfaction in junior secondary school students in Hong Kong: A 3-year longitudinal study" studied life satisfaction and the related socio-demographic and psychosocial correlates in junior secondary school students in Hong Kong. Regarding change in life satisfaction in Chinese aodlescents over time, there was a downward trend. Consistent with their predictions, adolescent life satisfaction was related to several demographic factors (gender, family intactness and economic disadvantage); family functioning and positive youth development also predicted adolescent life satisfaction over time.

To understand change and correlates of perceived family functioning in the adolescent years, Shek, Leung and Lu's paper "Perceived family life quality in junior secondary school students in Hong Kong" collected longitudinal data from junior secondary school students in Hong Kong. Perceived family functioning by adolescents gradually dropped over time. Consistent with the hypotheses, perceived family functioning was related to demographic factors (gender, family intactness, and economic disadvantage) and perceived family functioning.

Ip's paper: "Harmony as happiness? Comparing social harmonies in Hong Kong and China" is a study of the concept of social harmony between two Chinese societies: Hong Kong and China. It is a bold attempt to construct a concept of harmony that semantically approximates much of what is meant by happiness in Chinese societies. The concept of harmony was reconstructed by utilizing the measures and empirical data of harmony surveys conducted there. Meta-analysis of the harmony data and the related measures yielded the concepts of social harmony in the two places. A reconstructed concept is formed by integrating the extracted ones. The concept was compared with notions of wellbeing or happiness of these two societies to identify similarities and differences. The author argued that though semantically not identical, the two concepts share enough overlaps to warrant the view that a suitably developed measure of social harmony, no less than that of happiness, could be used as a possible alternative measure of the well-being of Chinese societies.

There are three papers that examine the well-being situation in Taiwan. The paper by Ip and Cheung "Probing folk happiness in Taiwan" presented a general picture of people's happiness in Taiwan. It explored the subjective well-being of its people based on a survey which involved a large sample. It included people's judgment about their satisfaction of life as a whole (global life satisfaction) or of specific aspects of life (domain satisfaction). Both the personal and societal aspects of people's reported life satisfaction were surveyed using a set of measures specifically constructed to be sensitive to the Taiwan social and political conditions. For example, cross-strait relationship was invoked as a measure to capture the taxing and often challenging Taiwan-China relationship. The meanings of the well-being findings were interpreted against the socio-political environment of Taiwan. The paper also examined the socio-demographic aspects, including gender, age, marital status, education, income, religion of the folk happiness of Taiwan. It is found that people has a modest level of life satisfaction.

The paper by Ku, Fox, Chang, Sun and Chen: "Cross-sectional and longitudinal associations of categories of physical activities with dimensions of subjective well-being in Taiwanese 
older adults" examined the impact of physical activities on the well-being of old people in Taiwan. However, physical activities that take place in different contexts may have differential impact on people's well-being. Basing on the data from two surveys drawn from Kaohsiung city in southern Taiwan, the study probed the well-being impact of three different kinds of physical activities: leisure-time; domestic, and work-related on elderly Kaohsiung residents. Results from the cross-sectional and longitudinal analyses revealed that that leisure-time physical activity was positively associated with 'physical', 'psychological', 'independence', 'learning and growth', and 'social' aspects of well-being. The authors basing on the data suggested that the association of physical activities with subjective well-being is contingent on the social context in which the activities took place. Another major finding of the study is that leisure-based physical activities are more conducive to well-being of the elderly.

Finally, The paper by Li and Tsai: "Is the easy life always the happiest? examining the association of convenience and well-being in Taiwan" explored the relationship between people's residential neighborhood and their life satisfaction in Taiwan. It examined whether neighborhood amenities and conveniences have impacts on people's well-being, health, and their satisfaction of life as a whole. Drawing on data from a separate survey undertaken by a separate entity, the authors investigated whether proximity to main roads, night markets and temples or proximity to smoky food stands etc. affected people's wellbeing. One major finding is that nearby annoyances is associated with people's lower neighborhood satisfaction, but is not associated with their lower life satisfaction in general. Meanwhile, air quality, and "peace and quietness" is reportedly well-being enhancing.

Theoretically, the papers included in this special issue provide some support to the Western theories and research findings. With reference to the three papers in Hong Kong, differences in quality of life in adolescents with and without economic disadvantage or non-intact family are generally in line with the Western findings; the relationship between positive youth development and family functioning is also consistent with the prediction of family ecological models. Interestingly, the drop in life satisfaction and family functioning across the early adolescent years deserves more research attention.

There are several methodological strengths of the studies in this special issue. First, with reference to Shek's (2010) comment that there are very few longitudinal family studies in the Chinese culture, three studies in Hong Kong were based on longitudinal research design. There are at least two reasons why longitudinal QOL and WB studies should be conducted. First, longitudinal studies can help researchers to understand changes in QOL and WB in Chinese people over time. Second, longitudinal studies can enable researchers to assess the predictors of QOL and WB in Chinese people and families. For example, without longitudinal studies, it would be quite impossible to examine how family intactness would impact on the development of children and adolescents.

The second methodological strength of the studies included in this special issue is that the sample size in the related studies was generally large, hence ensuring the power of the statistical tests performed. Finally, different conceptions and measures of QOL were adopted in the studies in this special issue. Besides individual QOL, family QOL was also involved.

\section{References}

Ip, P. K. (2009). Well-being of nations: A cross-cultural perspective. Social Indicators Research, 91, 1-3.

Shek, D. T. L. (2010). Quality of life of Chinese people in a changing world. Social Indicators Research, 95(3), 357-361. 
Shek, D. T. L. (2011). Quality of life in East Asia: The Case of Hong Kong. In K. C. Land, A. C. Michalos, \& M. J. Sirgy (Eds.), Handbook of social indicators and quality of life research (pp. 473-497). Netherlands: Springer Publishers.

Shek, D. T. L., Chan, Y. K., \& Lee, P. S. N. (2005). Quality of life in the global context: A Chinese response. Social Indicators Research, 71(1-3), 1-10.

The World Population Clock. (2013). Retrieved from website http://www.worldometers.info/worldpopulation/. 